\title{
Complete genome sequence of the gliding freshwater bacterium Fluviicola taffensis type strain (RW262 ${ }^{\mathrm{T}}$ )
}

\author{
Tanja Woyke ${ }^{1}$, Olga Chertkov ${ }^{1}$, Alla Lapidus ${ }^{1}$, Matt Nolan ${ }^{1}$, Susan Lucas ${ }^{1}$, Tijana Glavina Del \\ Rio $^{1}$, Hope Tice ${ }^{1}$, Jan-Fang Cheng ${ }^{1}$, Roxanne Tapia ${ }^{1,2}$, Cliff Han ${ }^{1,2}$, Lynne Goodwin ${ }^{1,2}$, Sam \\ Pitluck', Konstantinos Liolios ${ }^{1}$, Ioanna Pagani ${ }^{1}$, Natalia Ivanova ${ }^{1}$, Marcel Huntemann', \\ Konstantinos Mavromatis ${ }^{1}$, Natalia Mikhailova ${ }^{1}$, Amrita Pati ${ }^{1}$, Amy Chen ${ }^{3}$, Krishna \\ Palaniappan $^{3}$, Miriam Land ${ }^{1,4}$, Loren Hauser ${ }^{1,4}$, Evelyne-Marie Brambilla ${ }^{5}$, Manfred Rohde ${ }^{6}$, \\ Romano Mwirichia ${ }^{7}$, Johannes Sikorski ${ }^{5}$, Brian J. Tindall ${ }^{5}$, Markus Göker ${ }^{5}$, James Bristow ${ }^{1}$, \\ Jonathan A. Eisen ${ }^{1,7}$, Victor Markowitz ${ }^{4}$, Philip Hugenholtz ${ }^{1,9}$, Hans-Peter Klenk ${ }^{5}$, and Nikos \\ C. Kyrpides ${ }^{1 *}$ \\ ${ }^{1}$ DOE Joint Genome Institute, Walnut Creek, California, USA \\ ${ }^{2}$ Los Alamos National Laboratory, Bioscience Division, Los Alamos, New Mexico, USA \\ ${ }^{3}$ Biological Data Management and Technology Center, Lawrence Berkeley National \\ Laboratory, Berkeley, California, USA \\ ${ }^{4}$ Oak Ridge National Laboratory, Oak Ridge, Tennessee, USA \\ ${ }^{5}$ DSMZ - German Collection of Microorganisms and Cell Cultures GmbH, Braunschweig, \\ Germany \\ ${ }^{6} \mathrm{HZI}$ - Helmholtz Centre for Infection Research, Braunschweig, Germany \\ ${ }^{7}$ Jomo Kenyatta University of Agriculture and Technology, Kenya \\ ${ }^{8}$ University of California Davis Genome Center, Davis, California, USA \\ ${ }^{9}$ Australian Centre for Ecogenomics, School of Chemistry and Molecular Biosciences, The \\ University of Queensland, Brisbane, Australia \\ *Corresponding author: Nikos C. Kyrpides
}

Keywords: strictly aerobic, motile by gliding, Gram-negative, flexirubin-synthesizing, mesophilic, chemoorganotrophic, Cryomorphaceae, GEBA

\footnotetext{
Fluviicola taffensis O'Sullivan et al. 2005 belongs to the monotypic genus Fluviicola within the family Cryomorphaceae. The species is of interest because of its isolated phylogenetic location in the genome-sequenced fraction of the tree of life. Strain RW262 ${ }^{\top}$ forms a monophyletic lineage with uncultivated bacteria represented in freshwater 16S rRNA gene libraries. A similar phylogenetic differentiation occurs between freshwater and marine bacteria in the family Flavobacteriaceae, a sister family to Cryomorphaceae. Most remarkable is the inability of this freshwater bacterium to grow in the presence of $\mathrm{Na}^{+}$ions. All other genera in the family Cryomorphaceae are from marine habitats and have an absolute requirement for $\mathrm{Na}^{+}$ions or natural sea water. F. taffensis is the first member of the family Cryomorphaceae with a completely sequenced and publicly available genome. The 4,633,577 bp long genome with its 4,082 protein-coding and 49 RNA genes is a part of the Genomic Encyclopedia of Bacteria and Archaea project.
}

\section{Introduction}

Strain RW262 ${ }^{\mathrm{T}}$ (= DSM 16823 = NCIMB 13979) is the type strain of the species Fluviicola taffensis, which is the type species of the monotypic genus Fluviicola [1], affiliated with the family Cryomorphaceae [2]. The genus name is derived from the Latin words fluvius, meaning 'river' and -cola meaning 'inhabitant, dweller', yielding the NeoLatin word Fluviicola, the river dweller [1,3]. The species epithet is derived from the Neo-Latin word taffensis, referring to the place where the type strain has been isolated, the river Taff (Wales, UK) $[1,3]$. The family Cryomorphaceae belongs to the class Flavobacteria which contains many species that probably play an integral role for the flow of carbon and energy in the marine environment [4].

Flavobacteria are the major decomposers of highmolecular-mass organic matter in sea water [5]. 
Phylogenetically the family Cryomorphaceae is located between the families Flavobacteriaceae and Bacteroidaceae [2] and currently comprises the genera Brumimicrobium, Cryomorpha and Crocinitomix [2], Owenweeksia [6], Wandonia [7], Fluviicola [1] and Lishizhenia [8]. The family Cryomorphaceae exhibits the greatest degree of phenotypic similarity to the family Flavobacteriaceae [9] and includes species with a mostly rod-like to filamentous morphology; cells are usually nonmotile or move by gliding and often contain carotenoid pigments [1,2]. All members of the Cryomorphaceae are strictly aerobic or facultatively anaerobic (fermentative) with a chemoheterotrophic metabolism [1,2] and often have complex growth requirements for sea water salts, organic compounds as sole nitrogen sources, yeast extract and vitamins for growth [2]. To date no further isolates of $F$. taffensis have been reported. Here we present a summary classification and a set of features for F. taffensis RW262⿱一土 , together with the description of the complete genomic sequencing and annotation.

\section{Classification and features}

A representative genomic 16S rRNA sequence of $F$. taffensis RW262 ${ }^{\mathrm{T}}$ was compared using NCBI BLAST [10] under default settings (e.g., considering only the high-scoring segment pairs (HSPs) from the best 250 hits) with the most recent release of the Greengenes database [11] and the relative frequencies of taxa and keywords (reduced to their stem [12]) were determined, weighted by BLAST scores. The most frequently occurring genera were $\mathrm{Bru}$ mimicrobium (62.9\%) and Fluviicola (37.1\%) (3 hits in total). Among all other species, the one yielding the highest score was 'Brumimicrobium mesophilum' (DQ660382), which corresponded to an identity of $92.1 \%$ and an HSP coverage of $58.0 \%$. (Note that the Greengenes database uses the INSDC (= EMBL/NCBI/DDBJ) annotation, which is not an authoritative source for nomenclature or classification.) The most frequently occurring keywords within the labels of all environmental samples which yielded hits were 'lake' (9.1\%), 'tin' (3.4\%), 'microbi' (2.5\%), 'depth' (2.0\%) and 'tract' (1.7\%) (247 hits in total). The most frequently occurring keywords within those labels of environmental samples which yielded hits of a higher score than the highest scoring species were 'lake' (9.2\%), 'tin' (3.8\%), 'microbi' (2.3\%), 'depth' (2.0\%) and 'tract' (1.8\%) (169 hits in total). The most frequent key- word 'lake' may reflect the freshwater origin of strain RW262T, whereas the keywords 'tin' and 'depth' may allude to some until now unrecognized ecological features of $F$. taffensis.

Figure 1 shows the phylogenetic neighborhood of $F$. taffensis in a 16S rRNA based tree. The sequences of the two identical 16S rRNA gene copies in the genome differ by two nucleotides from the previously published 16S rRNA sequence (AF493694), which contains one ambiguous base call.

Strain RW262 ${ }^{\mathrm{T}}$ is strictly aerobic, Gram-negative, motile by gliding and flexirubin-pigmented [1]. Cells are flexible rods with rounded ends (Figure 2), 0.4-0.5 $\mu \mathrm{m}$ in diameter and 1.5-5.7 $\mu \mathrm{m}$ in length, with rare longer filaments of up to $51 \mu \mathrm{m}$ in length [1]. Growth occurs at $4^{\circ} \mathrm{C}$ and $20^{\circ} \mathrm{C}$, but not in the presence of $\mathrm{Na}^{+}$ions [1]. Growth of strain RW262 ${ }^{\mathrm{T}}$ at $4{ }^{\circ} \mathrm{C}$ is only weak, so that $F$. taffensis should not be considered to be psychrotolerant like the other members of the family $[1,2]$. Strain RW262 ${ }^{\mathrm{T}}$ is capable of DNA hydrolysis [1], is catalase positive but oxidase negative, able to catalyze the hydrolysis of arginine, aesculin or starch, whereas it weakly hydrolyzes gelatine [1]. It is negative for nitrate and nitrite reduction; indole production; $\beta$-galactosidase, urease and xylanase activity; hydrolysis of agar, arginine, aesculin and starch; and acid production from carbohydrates [1]. The strain is not able to utilize glucose, arabinose, mannose, mannitol, $\mathrm{N}$-acetylglucosamine, maltose, gluconate, caprate, adipate, malate, citrate or phenyl acetate [1]. However, within the genome are several genes for utilization of complex organic carbon compounds. The strain is resistant to chloramphenicol $(10 \mu \mathrm{g})$, streptomycin $(10 \mu \mathrm{g})$, and kanamycin (30 $\mu \mathrm{g})$ but susceptible to penicillin G (10 units), ampicillin $(10 \mu \mathrm{g})$, rifampicin $(5 \mu \mathrm{g})$ and tetracycline $(10 \mu \mathrm{g})$ [1].

\section{Chemotaxonomy}

The predominant cellular acid of strain RW262 was the branched-chain saturated fatty acid iso$\mathrm{C}_{15: 0}(44.2 \%)$ [1]. Unsaturated branched-chain fatty acids, straight-chain saturated and monounsaturated fatty acids occur only in lower amounts: $\mathrm{C}_{14: 0}$ (3.2\%), $\mathrm{C}_{15: 0}$ (7.5\%), $\mathrm{C}_{16: 0}$ (3.0\%), iso- $\mathrm{C}_{15: 1} \omega 10 \mathrm{c}(11.8 \%)$, iso- $\mathrm{C}_{16: 1} \omega 12 \mathrm{c}$ (4.9\%). Lipopolysaccharide hydroxy fatty acids constitute $20.4 \%$ of the total cellular fatty acids, mainly composed of iso- $\mathrm{C}_{17: 0}$ 3-он $(12.3 \%)$, iso- $\mathrm{C}_{15: 0}$ 3-он $(4.2 \%)$ and iso- $\mathrm{C}_{15: 02-\mathrm{OH}}(3.5 \%)$ [1]. 


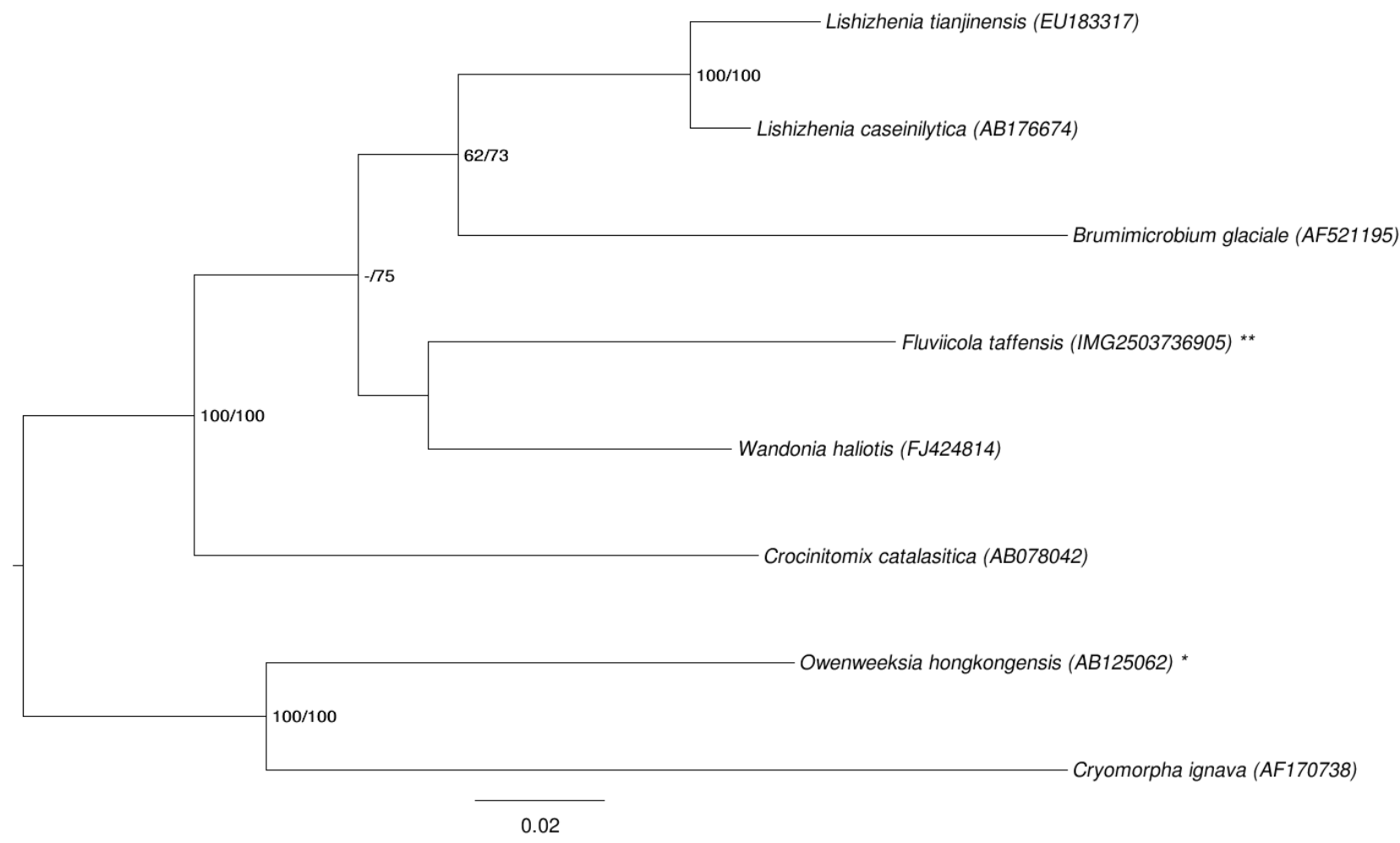

Figure 1. Phylogenetic tree highlighting the position of $F$. taffensis relative to the type strains of the other species within the family Cryomorphaceae. The tree was inferred from 1,429 aligned characters [13,14] of the $16 \mathrm{~S}$ rRNA gene sequence under the maximum likelihood (ML) criterion [15]. Rooting was done initially using the midpoint method [16] and then checked for its agreement with the current classification (Table 1). The branches are scaled in terms of the expected number of substitutions per site. Numbers adjacent to the branches are support values from $300 \mathrm{ML}$ bootstrap replicates [17] (left) and from 1,000 maximum parsimony bootstrap replicates [18] (right) if larger than $60 \%$. Lineages with type strain genome sequencing projects registered in GOLD [19] are labeled with one asterisk, those also listed as 'Complete and Published' (as well as the target genome) with two asterisks.

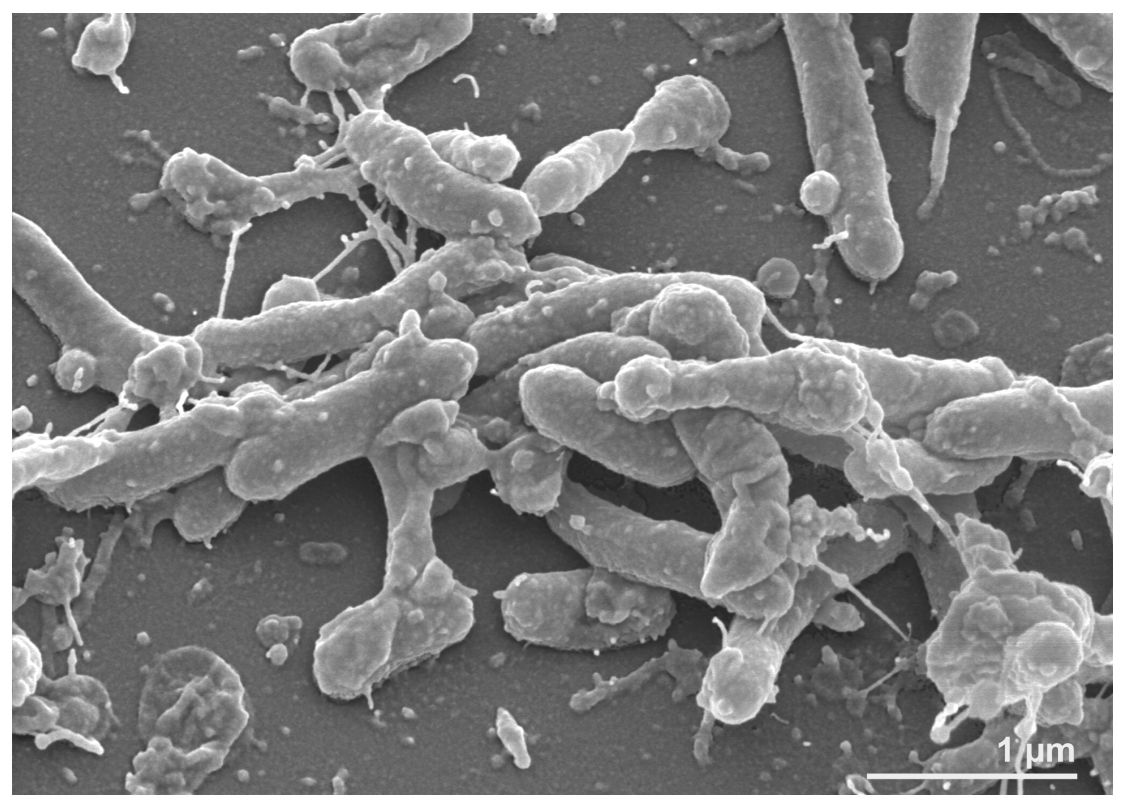

Figure 2. Scanning electron micrograph of $F$. taffensis $\mathrm{RW}^{262^{\top}}$ 
Table 1. Classification and general features of $F$. taffensis $\mathrm{RW} 262^{\top}$ according to the MIGS recommendations [20] and the NamesforLife database [21].

\begin{tabular}{|c|c|c|c|}
\hline MIGS ID & Property & Term & Evidence code \\
\hline & \multirow{8}{*}{ Current classification } & Domain Bacteria & TAS [22] \\
\hline & & Phylum Bacteroidetes & TAS [23] \\
\hline & & Class "Flavobacteria" & TAS [24] \\
\hline & & Order "Flavobacteriales" & TAS [25] \\
\hline & & Family Cryomorphaceae & TAS [2] \\
\hline & & Genus Fluviicola & TAS [1] \\
\hline & & Species Flaviicola taffensis & TAS [1] \\
\hline & & Type strain RW262 & TAS [1] \\
\hline & Gram stain & negative & TAS [1] \\
\hline & Cell shape & rod-shaped & TAS [1] \\
\hline & Motility & by gliding & TAS [1] \\
\hline & Sporulation & none & TAS [1] \\
\hline & Temperature range & $4^{\circ} \mathrm{C}-25^{\circ} \mathrm{C}$ & TAS [1] \\
\hline & Optimum temperature & $20^{\circ} \mathrm{C}$ & TAS [1] \\
\hline & Salinity & obligate $0 \%$ & TAS [1] \\
\hline \multirow[t]{3}{*}{ MIGS-22 } & Oxygen requirement & strict aerobe & TAS [1] \\
\hline & Carbon source & probably amino acids; unable to use carbohydrates & NAS \\
\hline & Energy metabolism & chemoorganotroph & TAS [1] \\
\hline MIGS-6 & Habitat & fresh water & TAS [1] \\
\hline MIGS-15 & Biotic relationship & free-living & NAS \\
\hline \multirow[t]{3}{*}{ MIGS-14 } & Pathogenicity & none & NAS \\
\hline & Biosafety level & 1 & TAS [26] \\
\hline & Isolation & fresh river water & TAS [1] \\
\hline MIGS-4 & Geographic location & River Taff near Cardiff, UK & TAS [1] \\
\hline MIGS-5 & Sample collection time & January 2000 & TAS [1] \\
\hline MIGS-4.1 & Latitude & 51.85 & TAS [1] \\
\hline MIGS-4.2 & Longitude & -2.32 & TAS [1] \\
\hline MIGS-4.3 & Depth & not reported & \\
\hline MIGS-4.4 & Altitude & sea level & NAS \\
\hline
\end{tabular}

Evidence codes - TAS: Traceable Author Statement (i.e., a direct report exists in the literature); NAS: Non-traceable Author Statement (i.e., not directly observed for the living, isolated sample, but based on a generally accepted property for the species, or anecdotal evidence). These evidence codes are from of the Gene Ontology project [27].

\section{Genome sequencing and annotation Genome project history}

This organism was selected for sequencing on the basis of its phylogenetic position [28], and is part of the Genomic Encyclopedia of Bacteria and Archaea project [29]. The genome project is deposited in the Genome On Line Database [19] and the complete genome sequence is deposited in GenBank. Sequencing, finishing and annotation were performed by the DOE Joint Genome Institute (JGI). A summary of the project information is shown in Table 2. 
Table 2. Genome sequencing project information

\begin{tabular}{|c|c|c|}
\hline MIGS ID & Property & Term \\
\hline MIGS-31 & Finishing quality & Finished \\
\hline MIGS-28 & Libraries used & $\begin{array}{l}\text { Tree genomic libraries: one } 454 \text { pyrosequence standard library, } \\
\text { one } 454 \text { PE library ( } 11 \text { kb insert size), one Illumina library }\end{array}$ \\
\hline MIGS-29 & Sequencing platforms & Illumina GAii, 454 GS FLX Titanium \\
\hline MIGS-31.2 & Sequencing coverage & $351.0 \times$ Illumina; $23.0 \times$ pyrosequence \\
\hline MIGS-30 & Assemblers & Newbler version 2.3, Velvet, phrap version SPS - 4.24 \\
\hline \multirow[t]{6}{*}{ MIGS-32 } & Gene calling method & Prodigal 1.4, GenePRIMP \\
\hline & INSDC ID & СР002542 \\
\hline & Genbank Date of Release & April 1, 2011 \\
\hline & GOLD ID & Gc01706 \\
\hline & NCBI project ID & 47603 \\
\hline & Database: IMG-GEBA & 2503707007 \\
\hline \multirow[t]{2}{*}{ MIGS-13 } & Source material identifier & DSM 16823 \\
\hline & Project relevance & Tree of Life, GEBA \\
\hline
\end{tabular}

\section{Growth conditions and DNA isolation}

F. taffensis RW262', DSM 16823, was grown in DSMZ medium 948 (Oxoid nutrient medium) [30] at $28^{\circ} \mathrm{C}$. DNA was isolated from $0.5-1 \mathrm{~g}$ of cell paste using JetFlex Genomic DNA Purification kit (GENOMED 600100) following the standard protocol as recommended by the manufacturer, but with additional $20 \mu \mathrm{l}$ proteinase $\mathrm{K}$ incubation (one hour) at $58^{\circ}$ for improved cell lysis. DNA is available through the DNA Bank Network [31].

\section{Genome sequencing and assembly}

The genome was sequenced using a combination of Illumina and 454 sequencing platforms. All general aspects of library construction and sequencing can be found at the JGI website [32]. Pyrosequencing reads were assembled using the Newbler assembler (Roche). The initial Newbler assembly consisting of 51 contigs in one scaffold was converted into a phrap [33] assembly by making fake reads from the consensus, to collect the read pairs in the 454 paired end library. Illumina GAii sequencing data (801.4 Mb) was assembled with Velvet [34] and the consensus sequences were shredded into $1.5 \mathrm{~kb}$ overlapped fake reads and assembled together with the 454 data. The 454 draft assembly was based on $164.9 \mathrm{Mb} 454$ draft data and all of the 454 paired end data. Newbler parameters are -consed -a 50 -1 350 -g -m -ml 20. The Phred/Phrap/Consed software package [33] was used for sequence assembly and quality assessment in the subsequent finishing process. After the shotgun stage, reads were assembled with parallel phrap (High Performance
Software, LLC). Possible mis-assemblies were corrected with gapResolution [32], Dupfinisher [35], or sequencing clones bridging PCR fragments with subcloning. Gaps between contigs were closed by editing in Consed, by PCR and by Bubble PCR primer walks (J.-F. Chang, unpublished). A total of 161 additional reactions and shatter libraries were necessary to close gaps and to raise the quality of the finished sequence. Illumina reads were also used to correct potential base errors and increase consensus quality using a software Polisher developed at JGI [36]. The error rate of the completed genome sequence was less than 1 in 100,000 . Together, the combination of the Illumina and 454 sequencing platforms provided 374.0 $\times$ coverage of the genome. The final assembly contained 232,904 pyrosequence and 44,902,395 Illumina reads.

\section{Genome annotation}

Genes were identified using Prodigal [37] as part of the Oak Ridge National Laboratory genome annotation pipeline, followed by a round of manual curation using the JGI GenePRIMP pipeline [38]. The predicted CDSs were translated and used to search the National Center for Biotechnology Information (NCBI) non-redundant database, UniProt, TIGR-Fam, Pfam, PRIAM, KEGG, COG, and InterPro databases. Additional gene prediction analysis and functional annotation was performed within the Integrated Microbial Genomes - Expert Review (IMG-ER) platform [39]. 


\section{Genome properties}

The genome consists of a 4,633,577 bp long chromosome with a $\mathrm{G}+\mathrm{C}$ content of $36.5 \%$ (Figure 3 and Table 3). Of the 4,131 genes predicted, 4,082 were protein-coding genes, and 49 RNAs; 49 pseudogenes were also identified. The majority of the protein-coding genes (55.0\%) were assigned a putative function while the remaining ones were annotated as hypothetical proteins. The distribution of genes into COGs functional categories is presented in Table 4.

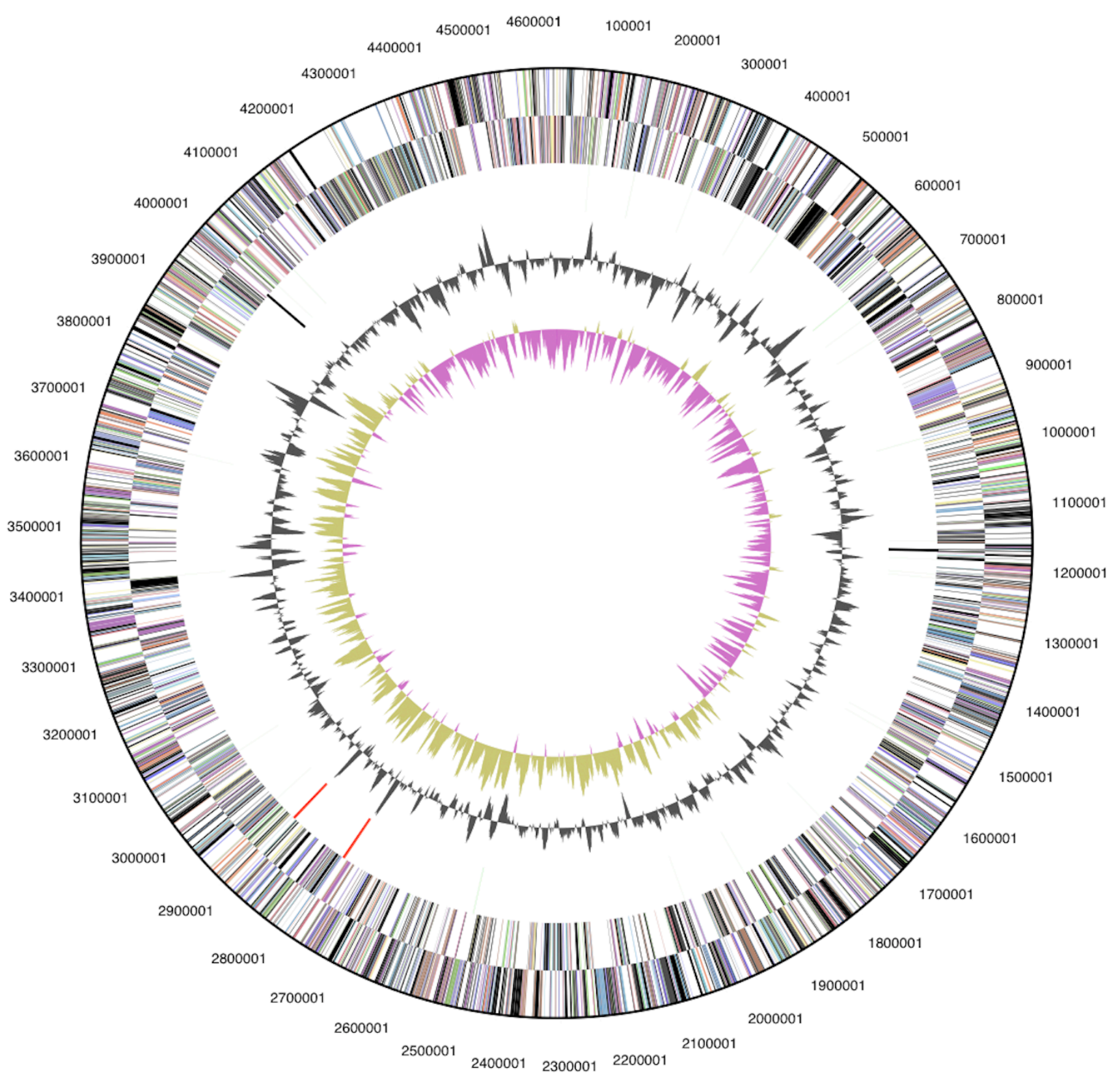

Figure 3. Graphical circular map of the genome. From outside to the center: Genes on forward strand (color by COG categories), Genes on reverse strand (color by COG categories), RNA genes (tRNAs green, rRNAs red, other RNAs black), GC content, GC skew. 
Table 3. Genome Statistics

\begin{tabular}{lrr}
\hline Attribute & Value & \% of Total \\
\hline Genome size (bp) & $4,633,577$ & $100.00 \%$ \\
DNA coding region (bp) & $4,192,830$ & $90.49 \%$ \\
DNA G+C content (bp) & $1,691,009$ & $36.49 \%$ \\
Number of replicons & 1 & \\
Extrachromosomal elements & 0 & \\
Total genes & 4,131 & $100.00 \%$ \\
RNA genes & 49 & $1.19 \%$ \\
rRNA operons & 2 & \\
Protein-coding genes & 4,082 & $98.81 \%$ \\
Pseudo genes & 49 & $1.19 \%$ \\
Genes with function prediction & 2,271 & $54.97 \%$ \\
Genes in paralog clusters & 532 & $12.88 \%$ \\
Genes assigned to COGs & 2,169 & $52.51 \%$ \\
Genes assigned Pfam domains & 2,420 & $58.58 \%$ \\
Genes with signal peptides & 1,331 & $32.22 \%$ \\
Genes with transmembrane helices & 911 & $22.05 \%$ \\
CRISPR repeats & 1 & \\
\hline
\end{tabular}

Table 4. Number of genes associated with the general COG functional categories

\begin{tabular}{crrl}
\hline Code & value & \%age & Description \\
\hline J & 168 & 6.0 & Translation, ribosomal structure and biogenesis \\
A & 0 & 0.0 & RNA processing and modification \\
K & 212 & 8.8 & Transcription \\
L & 137 & 5.7 & Replication, recombination and repair \\
B & 1 & 0.0 & Chromatin structure and dynamics \\
D & 22 & 0.9 & Cell cycle control, cell division, chromosome partitioning \\
Y & 0 & 0.0 & Nuclear structure \\
V & 57 & 2.4 & Defense mechanisms \\
T & 183 & 7.6 & Signal transduction mechanisms \\
M & 222 & 9.2 & Cell wall/membrane/envelope biogenesis \\
N & 8 & 0.3 & Cell motility \\
Z & 0 & 0.0 & Cytoskeleton \\
W & 0 & 0.0 & Extracellular structures \\
U & 42 & 1.8 & Intracellular trafficking, secretion, and vesicular transport \\
O & 106 & 4.4 & Posttranslational modification, protein turnover, chaperones \\
C & 117 & 4.9 & Energy production and conversion \\
G & 76 & 3.2 & Carbohydrate transport and metabolism \\
E & 136 & 5.7 & Amino acid transport and metabolism \\
F & 63 & 2.6 & Nucleotide transport and metabolism \\
H & 114 & 4.7 & Coenzyme transport and metabolism \\
I & 101 & 4.2 & Lipid transport and metabolism \\
P & 116 & 4.8 & Inorganic ion transport and metabolism \\
Q & 45 & 1.9 & Secondary metabolites biosynthesis, transport and catabolism \\
R & 286 & 11.9 & General function prediction only \\
S & 194 & 8.1 & Function unknown \\
- & 1,962 & 47.5 & Not in COGs \\
\hline & & & \\
\hline
\end{tabular}




\section{Acknowledgements}

We would like to gratefully acknowledge the help of Helga Pomrenke (DSMZ) for growing F. taffensis cultures. This work was performed under the auspices of the US Department of Energy Office of Science, Biological and Environmental Research Program, and by the University of California, Lawrence Berkeley National Laboratory under contract No. DE-AC02-05CH11231,

\section{References}

1. O'Sullivan LA, Rinna J, Humphreys G, Weightman AJ, Fry JC. Fluviicola taffensis gen. nov., sp. nov., a novel freshwater bacterium of the family Cryomorphaceae in the phylum 'Bacteroidetes'. Int I Syst Evol Microbiol 2005; 55:2189-2194. PubMed doi:10.1099/ijs.0.63736$\underline{0}$

2. Bowman JP, Mancuso Nichols C, Gibson JAE. Algoriphagus ratkowskyi gen. nov., sp. nov., Brumimicrobium glaciale gen. nov., sp. nov., Cryomorpha ignavagen. nov., sp. nov. and Crocinitomix catalasitica gen. nov., sp. nov., novel flavobacteria isolated from various polar habitats. Int J Syst Evol Microbiol 2003; 53:1343-1355. PubMed doi:10.1099/ijs.0.02553-0

3. Euzéby JP. List of bacterial names with standing in nomenclature: A folder available on the internet. Int I Syst Bacteriol 1997; 47:590-592. PubMed doi:10.1099/00207713-47-2-590

4. Kirchman DL. The ecology of CytophagaFlavobacteria in aquatic environments. FEMS Microbiol Ecol 2002; 39:91-100. PubMed

5. Cottrell MT, Kirchman DL. Natural assemblages of marine proteobacteria and members of the $\mathrm{Cy}$ tophaga-Flavobacter cluster consuming low - and high-molecular-weight dissolved organic matter. Appl Environ Microbiol 2000; 66:1692-1697. PubMed doi:10.1128/AEM.66.4.1692-1697.2000

6. Lau KWK, Ng CYM, Ren J, Lau SCL, Qian PY, Wong PK, Lau TC and WU M. Owenweeksia hongkongensis gen. nov., sp. nov., a novel marine bacterium of the phylum 'Bacteroidetes'. Int I Syst Evol Microbiol 2005; 55:1051-1057. PubMed doi:10.1099/ijs.0.63155-0

7. Lee DH, Choi EK, Moon SR, Ahn S, Lee YS, Jung JS, Jeon CO, Whang KS, Kahng HY. Wandonia haliotis gen. nov., sp. nov., a marine bacterium of the family Cryomorphaceae, phylum Bacteroidetes. Int / Syst Evol Microbiol 2010; 60:510-514. PubMed doi:10.1099/ijs.0.012674-0

8. Lau KWK, Ren J, Wai NLM, Qian PY, Wong PK, Wu M. Lishizhenia caseinilytica gen. nov., sp.
Lawrence Livermore National Laboratory under Contract No. DE-AC52-07NA27344, and Los Alamos National Laboratory under contract No. DE-AC0206NA25396, UT-Battelle and Oak Ridge National Laboratory under contract DE-AC05-000R22725, as well as German Research Foundation (DFG) INST 599/1-2.

nov., a marine bacterium of the phylum Bacteroidetes. Int J Syst Evol Microbiol 2006; 56:23172322. PubMed doi:10.1099/ijs.0.64415-0

9. Bernardet JF, Nakagawa Y, Holmes B. Proposed minimal standards for describing new taxa of the family Flavobacteriaceae and emended description of the family. Int J Syst Evol Microbiol 2002; 52:1049-1070. PubMed doi:10.1099/ijs.0.02136$\underline{0}$

10. Altschul SF, Gish W, Miller W, Myers EW, Lipman DJ. Basic local alignment search tool. J Mol Biol 1990; 215:403-410. PubMed

11. DeSantis TZ, Hugenholtz P, Larsen N, Rojas M, Brodie EL, Keller K, Huber T, Dalevi D, Hu P, Andersen GL. Greengenes, a chimera-checked $16 \mathrm{~S}$ rRNA gene database and workbench compatible with ARB. Appl Environ Microbiol 2006; 72:5069-5072. PubMed doi:10.1128/AEM.03006-05

12. Porter MF. An algorithm for suffix stripping. Program: electronic library and information systems 1980; 14:130-137.

13. Lee C, Grasso C, Sharlow MF. Multiple sequence alignment using partial order graphs. Bioinformatics 2002; 18:452-464. PubMed doi:10.1093/bioinformatics/18.3.452

14. Castresana J. Selection of conserved blocks from multiple alignments for their use in phylogenetic analysis. Mol Biol Evol 2000; 17:540-552. PubMed

15. Stamatakis A, Hoover P, Rougemont J. A rapid bootstrap algorithm for the RAxML web-servers. Syst Biol 2008; 57:758-771. PubMed doi:10.1080/10635150802429642

16. Hess PN, De Moraes Russo CA. An empirical test of the midpoint rooting method. Biol / Linn SOC Lond 2007; 92:669-674. doi:10.1111/j.10958312.2007.00864.x

17. Pattengale ND, Alipour M, Bininda-Emonds ORP, Moret BME, Stamatakis A. How many bootstrap replicates are necessary? Lect Notes Comput Sci 
2009; 5541:184-200. doi:10.1007/978-3-642$\underline{02008-7 \quad 13}$

18. Swofford DL. PAUP*: Phylogenetic Analysis Using Parsimony (*and Other Methods), Version 4.0 b10. Sinauer Associates, Sunderland, 2002.

19. Liolios K, Chen IM, Mavromatis K, Tavernarakis N, Hugenholtz P, Markowitz VM, Kyrpides NC. The Genomes On Line Database (GOLD) in 2009: status of genomic and metagenomic projects and their associated metadata. Nucleic Acids Res 2010; 38:D346-D354. PubMed doi:10.1093/nar/gkp848

20. Field D, Garrity G, Gray T, Morrison N, Selengut J, Sterk P, Tatusova T, Thomson N, Allen MJ, Angiuoli $\mathrm{SV}$, et al. The minimum information about a genome sequence (MIGS) specification. Nat Biotechnol 2008; 26:541-547. PubMed doi:10.1038/nbt1360

21. Garrity G. NamesforLife. BrowserTool takes expertise out of the database and puts it right in the browser. Microbiol Today 2010; 37:9.

22. Woese CR, Kandler O, Wheelis ML. Towards a natural system of organisms: proposal for the domains Archaea, Bacteria, and Eucarya. Proc Natl Acad Sci USA 1990; 87:4576-4579. PubMed doi:10.1073/pnas.87.12.4576

23. Garrity GM, Holt JG. The Road Map to the Manual. In: Garrity GM, Boone DR, Castenholz RW (eds), Bergey's Manual of Systematic Bacteriology, Second Edition, Volume 1, Springer, New York, 2001, p. 119-169.

24. Ludwig W, Euzeby J, Whitman WG. Draft taxonomic outline of the Bacteroidetes, Planctomycetes, Chlamydiae, Spirochaetes, Fibrobacteres, Fusobacteria, Acidobacteria, Verrucomicrobia, Dictyoglomi, and Gemmatimonadetes. http://www.bergeys.org/outlines/Bergeys_Vol_4 Outline.pdf

25. Garrity GM, Holt JG. 2001. Taxonomic outline of the Archaea and Bacteria, p. 155-166. In: Garrity GM, Boone DR, Castenholz RW (ed), Bergey's Manual of Systematic Bacteriology, 2nd ed, vol. 1. Springer, New York.

26. BAuA. 2010, Classification of bacteria and archaea in risk groups. http://www.baua.de TRBA 466, p. 89.

27. Ashburner M, Ball CA, Blake JA, Botstein D, Butler H, Cherry JM, Davis AP, Dolinski K, Dwight SS, Eppig JT, et al. Gene Ontology: tool for the unification of biology. Nat Genet 2000; 25:25-29. $\underline{\text { PubMed doi:10.1038/75556 }}$
28. Klenk HP, Göker M. En route to a genome-based classification of Archaea and Bacteria? Syst Appl Microbiol 2010; 33:175-182. PubMed doi:10.1016/j.syapm.2010.03.003

29. Wu D, Hugenholtz P, Mavromatis K, Pukall R, Dalin E, Ivanova NN, Kunin V, Goodwin L, Wu $M$, Tindall BJ, et al. A phylogeny-driven genomic encyclopaedia of Bacteria and Archaea. Nature 2009; 462:1056-1060. PubMed doi:10.1038/nature08656

30. List of growth media used at DSMZ: http://www.dsmz.de/microorganisms/media_list.p $\underline{h p}$

31. Gemeinholzer B, Dröge G, Zetzsche H, Haszprunar G, Klenk HP, Güntsch A, Berendsohn WG, Wägele JW. The DNA Bank Network: the start from a German initiative. Biopreservation and Biobanking 2011; 9:51-55.

doi:10.1089/bio.2010.0029

32. JGI website. http://www.jgi.doe.gov

33. The Phred/Phrap/Consed software package. http://www.phrap.com

34. Zerbino DR, Birney E. Velvet: algorithms for de novo short read assembly using de Bruijn graphs. Genome Res 2008; 18:821-829. PubMed doi:10.1101/gr.074492.107

35. Han C, Chain P. Finishing repeat regions automatically with Dupfinisher. In: Proceeding of the 2006 international conference on bioinformatics \& computational biology. Arabnia HR, Valafar $\mathrm{H}$ (eds), CSREA Press. June 26-29, 2006: 141-146.

36. Lapidus A, LaButti K, Foster B, Lowry S, Trong S, Goltsman E. POLISHER: An effective tool for using ultra short reads in microbial genome assembly and finishing. AGBT, Marco Island, FL, 2008.

37. Hyatt D, Chen GL, LoCascio PF, Land ML, Larimer FW, Hauser LJ. Prodigal: prokaryotic gene recognition and translation initiation site identification. BMC Bioinformatics 2010; 11:119. PubMed doi:10.1186/1471-2105-11-119

38. Pati A, Ivanova NN, Mikhailova N, Ovchinnikova G, Hooper SD, Lykidis A, Kyrpides NC. GenePRIMP: a gene prediction improvement pipeline for prokaryotic genomes. Nat Methods 2010; 7:455-457. PubMed doi:10.1038/nmeth.1457

39. Markowitz VM, Ivanova NN, Chen IMA, Chu K, Kyrpides NC. IMG ER: a system for microbial genome annotation expert review and curation. Bioinformatics 2009; 25:2271-2278. PubMed doi:10.1093/bioinformatics/btp393 
